Draft Version February 25, 2021

Typeset using $\mathrm{LAT}_{\mathrm{E}} \mathrm{X}$ twocolumn style in AASTeX63

\title{
Tumbling Dice: Radio Constraints on the Presence of Circumstellar Shells around Type Ia Supernovae with Impact Near Maximum Light
}

\author{
Chelsea E. Harris (D), ${ }^{1}$ Laura Chomiuk (D) ${ }^{1}$ and Peter. E. Nugent (iD ${ }^{2,3}$ \\ ${ }^{1}$ Center for Data Intensive and Time Domain Astronomy, Department of Physics and Astronomy, Michigan State University, East \\ Lansing, MI 48824, USA \\ ${ }^{2}$ Lawrence Berkeley National Laboratory, 1 Cyclotron Road, MS 50B-4206, Berkeley, CA 94720, USA \\ ${ }^{3}$ Department of Astronomy, University of California, Berkeley, CA 94720-3411, USA
}

(Received January 29, 2021; Accepted February 25, 2021)

Submitted to ApJ

\begin{abstract}
The progenitors of Type Ia supernovae (SNeIa) are debated, particularly the evolutionary state of the binary companion that donates mass to the exploding carbon-oxygen white dwarf. In previous work, we presented hydrodynamic models and optically thin radio synchrotron light-curves of SNe Ia interacting with detached, confined shells of CSM, representing CSM shaped by novae. In this work, we extend these light-curves to the optically thick regime, considering both synchrotron self-absorption and free-free absorption. We obtain simple formulae to describe the evolution of optical depth seen in the simulations, allowing optically thick light-curves to be approximated for arbitrary shell properties. We then demonstrate the use of this tool by interpreting published radio data. First, we consider the non-detection of PTF11kx - an SN Ia known to have a detached, confined shell - and find that the nondetection is consistent with current models for its CSM, and that observations at a later time would have been useful for this event. Secondly, we statistically analyze an ensemble of radio non-detections for SNe Ia with no signatures of interaction, and find that shells with masses $\left(10^{-4}-0.3\right) M_{\odot}$ located $\left(10^{15}-10^{16}\right) \mathrm{cm}$ from the progenitor are currently not well constrained by radio datasets, due to their dim, rapidly-evolving light-curves.
\end{abstract}

Keywords: Type Ia supernovae(1728) — Circumstellar gas(238) — Shocks(2086)

\section{INTRODUCTION}

Thermonuclear Type Ia supernovae (SNeIa) are one of the most mature and precise cosmological tools in modern astronomy, and have revealed the accelerating expansion of the universe (Riess et al. 1998; Perlmutter et al. 1999). SNe Ia are the explosion of a carbon-oxygen white dwarf that has merged with or accreted mass from a companion star. However, we

currently remain ignorant of the identity of the companion star, which affects the timescale of explosion, explosion trigger, properties of the white dwarf at time of explosion, and local environment.

Corresponding author: $\mathrm{CEH}$

harr1561@msu.edu
It has long been recognized that characterizing the circumstellar material (CSM) around SNe Ia constrains the nature of their companions (Branch et al. 1995). For example, main sequence and red giant companions of the "single-degenerate" channel will have winds. Growth of the white dwarf happens through accretion, either Roche-lobe overflow or directly from the companion wind (i.e., a symbiotic system). Instabilities in this mass-transfer (e.g., novae) can create dense shells of hydrogen-rich CSM. In contrast, the "doubledegenerate" channel, where explosion is triggered by the merger of two white dwarfs, is expected to have a clean environment.

Radio observations are sensitive probes of the CSM around $\mathrm{SNe}$, as synchrotron emission is produced when the SN blast wave shocks surrounding gas, accelerates electrons to relativistic speeds, and amplifies the magnetic field in the shocked region (Chevalier 1982). This 
emission will be subject to absorption, and of particular relevance to this work is the absorption caused by the CSM itself: within the shock region, radio emission is affected by synchrotron self-absorption, and radio emission emerging from the shock region is further subject to free-free absorption from outlying, unshocked CSM. Despite extensive observations of SNe Ia at radio wavelengths, there are no published radio detections of SNeIa to date, even for those known to be interacting. Upper limits on radio luminosity imply that the CSM around typical SNe Ia is substantially lower density than observed around most core-collapse SNe, assuming the CSM is a continuous medium like a wind (Weiler et al. 2002; Pérez-Torres et al. 2014; Chomiuk et al. 2016; Lundqvist et al. 2020).

In recent decades, the picture of SN Ia environments and the single-degenerate channel has become muddied by the discovery of what was long-sought: SNe Ia with signatures of hydrogen (from CSM interaction) in their spectra, dubbed SNe Ia-CSM by Silverman et al. (2013). SNe Ia-CSM can be broken into two groups. The first and most common are events like SN 2005gj, which were historically grouped with the canonical CSM interaction class of SNe IIn but have distinct underlying SN Ia features. Radio non-detections are expected for such events since light at radio frequencies will be totally absorbed by the outlying CSM that has not yet been shocked. The other case is more rare, where an SN Ia transforms from a normal event into an interacting event (which we for shorthand call SNe Ia;n, Harris et al. 2018). The prototype is PTF11kx (Dilday et al. 2012), though SN 2002ic may also have been an SN Ia;n (Wood-Vasey et al. 2004). A search for more instances of SNe Ia;n discovered interaction in SN 2015cp (Graham et al. 2019). The CSM of SNe Ia;n may be shaped by nova outbursts or other instabilities in the mass-transfer process that sweep any existing material into a distant shell.

SNe Ia;n are of particular interest because they are so disruptive to our current theoretical understanding and abilities, and to SN Ia observational traditions yet they are a clear path forward to understanding the single-degenerate channel. They disrupt our theoretical understanding because novae should interrupt the mass growth of the carbon-oxygen white dwarf (see, e.g., the discussion and references in Branch et al. 1995). Yet the CSM mass observed for PTF11kx (Graham et al. 2017) was too low to have come from an expelled common envelope of the double-degenerate scenario (Livio \& Riess 2003). They disrupt theoretical ability because the wellestablished tools for interpreting interaction with a wind or other continuous medium cannot be applied (Chevalier 1982). SNe Ia;n are furthermore extremely diffi- cult to detect via traditional SN Ia observation methods, which only cover the phase near maximum light during which the CSM will not be visible (even in spectra, as was the case for SN 2015cp). Furthermore, the interaction may be very short-lived, eluding even observations at late times - and the fact that the time between mass ejection and supernova is unknown means the location of the shell is unknown and potentially random. Finally, as is the case with the SN IIn-like events, SNe Ia;n come from the rare "shallow silicon" or "SN 1991T-like" subgroup of SNe Ia, making the chance of discovery even smaller since SN Ia surveys usually attempt to recreate the underlying distribution of SN Ia properties. Despite all of these difficulties, SNe Ia;n are the clearest path forward to understanding the single-degenerate channel, because we can constrain the ejecta properties from pre-interaction data to alleviate degeneracies in the interaction modelling, and because the CSM mass is too low to be explained by a double-degenerate origin (as aforementioned).

The potential for SNe Ia;n to illuminate SN Ia progenitors motivates the alleviation of the theoretical obstacles facing their study. Harris et al. (2016, Paper I) modeled SNe Ia interacting with low-mass, confined shells of CSM in the months following maximum light. The optically thin radio light-curves from these models were then studied, and a parameterization was created to allow for light-curves to be created for an arbitrary CSM shell configuration. These light-curves can be used to limit CSM shell properties from radio non-detections particularly for the very thin, low-mass shells expected from single nova eruptions, distant shells that will be very low density, or radio observations taken after interaction has ended (Harris et al. 2018; Cendes et al. 2020; Pellegrino et al. 2020).

However, there is a sizeable sample of SNe Ia with radio observations near maximum light, probing shells at a distance $r \sim\left(10^{15}-10^{16}\right) \mathrm{cm}$ (Chomiuk et al. 2016), and the use of this dataset is currently limited by the optically thin assumption, which is only applicable to shells of density $\lesssim 10^{-17} \mathrm{~g} \mathrm{~cm}^{-3}$ or after the shock has crossed the shell. In order to study interaction within $r \sim 10^{16} \mathrm{~cm}$ and to incorporate lower-frequency observations, the optically thin light-curves of Paper I must be extended into the regime of synchrotron self-absorption and external free-free absorption, which is our aim for this work. With absorption accounted for, we can use the radio sample to constrain the presence of nova-like shells around SNe Ia for higher shell masses than was previously possible.

This work is organized as follows. In $\S 2$, we summarize the main results of Paper I for the reader's conve- 
nience. We then present the method for modifying the optically thin luminosity by the photon escape fraction to obtain a light-curve with absorption in $\S 3$. We account for synchrotron self-absorption $\left(\tau_{\text {ssa }}\right)$ and free-free $\left(\tau_{\mathrm{ff}}\right)$ absorption. In $\S 4$ we show the evolution of optical depth as calculated directly from the hydrodynamic models. The creation of optically-thick light-curves for an arbitrary shell configuration without the need for hydrodynamic simulations is enabled by the parameterization of $\tau_{\text {ssa }}(t)$ and $\tau_{\mathrm{ff}}(t)$ that we give in $\S 5$. In $\S 6$ we show how this parameterization can be applied to the planning and interpretation of observations. First, we look at the radio non-detection of PTF11kx, an SN Ia known to interact with a confined, detached shell of CSM. We then perform a statistical analysis of radio non-detections of SNe Ia near maximum light to derive the maximum allowed fraction of SNe Ia that can host confined, detached shells.

\section{SUMMARY OF PAPER I}

Paper I presented a suite of one-dimensional hydrodynamic models of a typical SN Ia interacting with a low-mass, confined shell of CSM. This section provides a brief summary of the Paper I results and reiterates its limitations for the reader's convenience.

The SN Ia ejecta have mass $M_{\mathrm{ej}}=1.38 M_{\odot}$ and energy $E_{\text {ej }}=10^{51} \mathrm{erg}$. Before impact with the CSM shell, they are in free expansion. This is equivalent to assuming that any CSM within the detached shell is too low density to affect the dynamics of the ejecta. The ejecta mass-density profile is assumed to have a broken power-law structure with $\rho_{\mathrm{ej}} \propto r^{-1}$ in the inner regions $\left(v \lesssim 10,000 \mathrm{~km} \mathrm{~s}^{-1}\right)$ and $\rho_{\mathrm{ej}} \propto r^{-10}$ in the outer regions.

The CSM is assumed to be confined to a constantdensity shell with density $\rho_{\mathrm{csm}}$ between radii $R_{\text {in }}$ and $\left(1+f_{R}\right) R_{\text {in }}$. The parameter $f_{R} \in[0.1,1]$ is called the "fractional width" of the shell, since

$$
f_{R}=\Delta R / R_{\text {in }},
$$

where $\Delta R$ is the width of the shell.

The ejecta impact the CSM at time $t_{\text {imp }}$ after explosion. The time of impact is related to $R_{\text {in }}$ through $\mathrm{Pa}-$ per I Equation 5,

$$
\begin{aligned}
R_{\mathrm{in}}= & \left(1.47 \times 10^{16} \mathrm{~cm}\right)\left(\frac{t_{\mathrm{imp}}}{100 \text { days }}\right)^{0.7} \\
& \times\left(\frac{\rho_{\mathrm{csm}}}{10^{-18} \mathrm{~g} \mathrm{~cm}^{-3}}\right)^{-0.1} .
\end{aligned}
$$

This scaling ensures that the density ratio between the CSM and ejecta at the point and time of first contact is fixed to 0.33 , which defines the "fiducial model set."
The models are invalid when (1) $R_{\text {in }} / t_{\text {imp }}>$ $45,000 \mathrm{~km} \mathrm{~s}^{-1}$, i.e., they imply an unphysically large ejecta speed, (2) $R_{\mathrm{in}} / t_{\mathrm{imp}}<10,000 \mathrm{~km} \mathrm{~s}^{-1}$, i.e., interaction is with the inner ejecta, or $(3) \rho_{\text {csm }}>10^{-14} \mathrm{~g} \mathrm{~cm}^{-3}$ where cooling and photon trapping are likely to be important, i.e., the adiabatic assumption does not hold. At a given $R_{\text {in }}$, the first constraint places a lower limit on the allowed CSM densities, whereas the second two place upper limits on the CSM density. These limitations are summarized in Figure 1 of Paper I.

The hydrodynamics are evolved assuming adiabatic evolution using the one-dimensional Lagrangian solver of SEDONA (Roth \& Kasen 2015). The hydrodynamic behavior of this system is as follows. Initially, the shock "ramps" up in the CSM - energy density grows, as does the width of the shock region. Before it can reach the self-similar limit, the forward shock reaches the edge of the CSM shell - the "end" of interaction. The hot, accelerated CSM is uncontained by any external material and therefore rapidly expands - a rarefaction wave crosses back toward the ejecta. The energy density plummets.

Paper I assumes the relativistic electron population in the shocked gas is distributed as

$$
n_{e}(E) d E=C_{E} E^{-p} d E
$$

where $E$ is the electron energy, and $p=3$ is assumed. The normalization factor is determined by assuming that the energy density in relativistic electrons is $10 \%$ of the total shocked gas energy density, i.e. $\epsilon_{e}=0.1$.

Paper I shows that the fiducial model set defines a family of optically-thin light-curves. The light-curves rise while the shock is in the CSM and therefore peak at the time the shock reaches the outer edge of the CSM shell. For this reason, the time the shock reaches the outer edge of the CSM is denoted $t_{p}$. Paper I Equation 7 gives

$$
t_{p} / t_{\text {imp }}=0.983\left(1+f_{R}\right)^{1.28}
$$

which can be used to produce an expression for the evolution of the forward shock radius $\left(R_{f}\right)$, since $R_{\text {out }} / R_{\text {in }}=\left(1+f_{R}\right)$,

$$
R_{f} / R_{\text {in }}=1.013\left(t / t_{\text {imp }}\right)^{0.781} .
$$

The peak luminosity scales like

$$
\begin{aligned}
\mathcal{L}_{\nu, \text { thin }, p} \propto & \epsilon_{e}^{2} \epsilon_{B} \nu^{-1} \rho_{\mathrm{csm}}^{8 / 7} R_{\mathrm{in}}^{3 / 7} \\
& \times\left[1-\left(1+f_{R}\right)^{-1.28}\right],
\end{aligned}
$$

as can be seen in Paper I Equations 11 and 37, where $\epsilon_{B}$ is the ratio of the magnetic field energy density density to the gas energy density and is typically assumed to be $\epsilon_{B}=0.1$ though in this work we leave it as a free parameter. 
The shape of the light-curves is described by an asymptotic rise (Paper I Equation 10) followed by a complex decline. The decline is described by the $f_{R^{-}}$ dependent time it takes the light-curve to reach characteristic fractions of the peak luminosity (Paper I Table 1 and Equation 12).

\section{ESCAPE FRACTION OF PHOTONS FROM A THIN SPHERICAL SHELL}

In this work we consider the absorption of radio emission from synchrotron self-absorption in the emitting region itself as well as external absorption by the free-free (Bremsstrahlung) process in the external, unshocked CSM. The strategy for obtaining optically thick radio light-curves from the optically thin light-curves parameterized in Paper I is to simply find the escape fraction of radio photons, the ratio of the optically thick to optically thin luminosity.

The expression for the escape fraction depends on the geometry of the emitting and absorbing gasses. In our case, the emitting (and self-absorbing) region is a thin, spherical shell. The external, absorbing medium is also a thin, spherical shell and only exists before the forward shock overtakes the edge of the shell.

Weiler et al. (1990) provide an expression for correcting optically thin luminosity for internal absorption in the emitting medium,

$$
\mathcal{L}=\mathcal{L}_{\text {thin }}\left(\frac{1-e^{-\tau}}{\tau}\right)
$$

which is the calculation for a planar slab geometry and $\tau$ is the optical depth of the slab along the line of sight. In Appendix A, we show that the full solution for a thin shell geometry has the same asymptotic behavior as the slab approximation so long as one uses an appropriate expression for $\tau$. Given the synchrotron self-absorption extinction coefficienct $\left(\alpha_{\text {ssa }}\right)$ and the volume-to-surfacearea ratio of the emitting sphere $(\widetilde{\Delta r})$, the appropriate $\tau$ to capture the effect of synchrotron self-absorption is

$$
\tau_{\mathrm{ssa}}=4 \alpha_{\mathrm{ssa}} \widetilde{\Delta r}
$$

such that the escape fraction in the absence of an external absorbing medium can be approximated by

$$
\mathcal{L}_{\nu} / \mathcal{L}_{\nu, \text { thin }}=\frac{1-e^{-\tau_{\mathrm{ssa}}}}{\tau_{\mathrm{ssa}}}
$$

as derived in Appendix A.1. In the presence of an absorbing medium with extinction coefficient $\alpha_{\mathrm{ff}}$ and radial width $\Delta r_{\text {ext }}$, we take the free-free optical depth to be

$$
\tau_{\mathrm{ff}}=\alpha_{\mathrm{ff}} \Delta r_{\mathrm{ext}}
$$

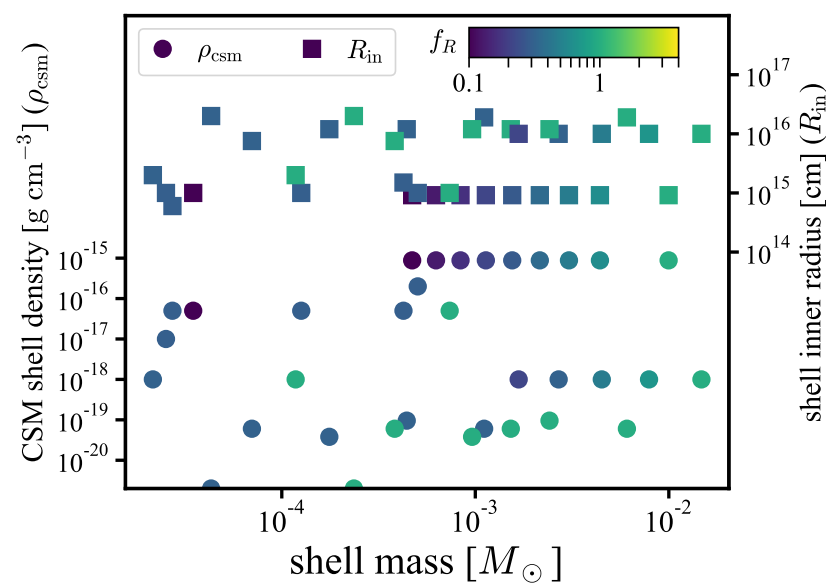

Figure 1. Summary of the CSM shell properties for the simulations presented in this work. Circles show the CSM density (left axis) and squares represent the inner radius (right axis), and color illustrates the shell width (color bar). Axis limits are set to separate the density and radius points.

and approximate the escape fraction as

$$
\mathcal{L}_{\nu} / \mathcal{L}_{\nu, \text { thin }}=\frac{1-e^{-\tau_{\mathrm{ssa}}}}{\tau_{\mathrm{ssa}}} e^{-\tau_{\mathrm{ff}}}
$$

as discussed in Appendix A.2. As noted in the appendices, the error incurred by using these approximations in lieu of the exact integral depends on the extent of the media and their optical depth but is typically small.

Thus, the goal of this work is to determine, from the simulations, the time evolution of $\alpha_{\mathrm{ssa}} \widetilde{\Delta r}$ and $\alpha_{\mathrm{ff}} \Delta r_{\mathrm{ext}}$.

\section{CALCULATION OF OPTICAL DEPTH FROM SIMULATIONS}

In this section we describe how we calculate the optical depths $\tau_{\text {ssa }}$ and $\tau_{\text {ff }}$ from hydrodynamic models. Figure 1 shows the CSM shell properties of the models, which cover a range of shell masses through variations in the shell location, extent, and density.

First we must calculate the extinction coefficient of synchrotron self-absorption, $\alpha_{\text {ssa }}$, in each shocked resolution element for each simulation snapshot. We perform these calculations with rad_tools.SynchrotronCalculator of csmpy. ${ }^{1}$

The synchrotron extinction coefficient $\left(\alpha_{\text {ssa }}\right)$ in each resolution element of the simulation is calculated accord-

\footnotetext{
${ }^{1}$ https://github.com/chelseaharris/csmpy
} 
ing to the equation in Rybicki \& Lightman (1979),

$$
\begin{aligned}
\alpha_{\mathrm{ssa}}(\nu)= & \nu^{-(p+4) / 2} \frac{\sqrt{3} q_{e}^{3}}{8 \pi m_{e}}\left(\frac{3 q_{e}}{2 \pi m_{e}^{3} c^{5}}\right)^{p / 2} \\
& \times C_{E}(2 B / \pi)^{(p+2) / 2} \\
& \times \Gamma\left(\frac{3 p+2}{12}\right) \Gamma\left(\frac{3 p+22}{12}\right),
\end{aligned}
$$

where $p=3$ is the electron distribution power-law index, $q_{e}$ is the electron charge, $m_{e}$ the electron mass, $c$ the speed of light, $C_{E}$ is the normalization of the electron distribution (Equation 3, $B=\sqrt{8 \pi \epsilon_{B} u_{\text {gas }}}$ the magnetic field strength (the factor of $2 / \pi$ multiplying $B$ in $\alpha_{\text {ssa }}$ accounts for the pitch angle term as in $\mathrm{Pa}-$ per I), and $\Gamma$ is the gamma function (calculated using scipy.special.gamma).

Equation 11 assumes a constant extinction coefficient in the self-absorbing shell. In reality, especially after the shock crosses the shell and it begins to expand, the extinction coefficient may be different across the shocked gas. The representative $\alpha$ we use in our optical depth calculations is the radial average value,

$$
\left\langle\alpha_{\mathrm{ssa}}\right\rangle=\frac{\sum_{k} \alpha_{\mathrm{ssa}, k} d r_{k}}{\sum_{k} d r_{k}},
$$

where $k$ indicates the index of a resolution element in the shock. We exclude the five resolution elements closest to the contact discontinuity in our calculation of $\left\langle\alpha_{\mathrm{ssa}}\right\rangle$ because mass-density is a factor in the $\alpha_{\text {ssa }}$ calculations and is incorrect near the contact discontinuity due to the unaddressed Rayleigh-Taylor instability, as noted, e.g., in Chevalier (1982).

The representative shell thickness $\widetilde{\Delta r}$ (Equation A6) can be computed directly from the contact discontinuity radius $\left(r_{1}\right)$ and the forward shock radius $\left(r_{2}\right)$.

Thus for each time snapshot of the simulation we can determine

$$
\tau_{\mathrm{ssa}}=4\left\langle\alpha_{\mathrm{ssa}}\right\rangle \widetilde{\Delta r}
$$

The free-free extinction coefficient $\left(\alpha_{\mathrm{ff}}\right)$ must describe the preshock CSM, which we assume is hydrogen rich, isothermal, constant density, and fully ionized by the radiation field of the shock. Therefore, although there are many resolution elements of preshock CSM in the hydrodynamic simulation, for the purposes of radiation transport it is one-zone model. The extinction coefficient is calculated using the formulae in Rybicki \& Lightman (1979) via the rad_tools. BremCalculator. calc_al_BB function of csmpy which assumes the electrons are thermally distributed and uses the gaunt factors calculated by van Hoof et al. (2014). Extinction by the CSM is in the Rayleigh-Jeans limit and the formula used is

$$
\alpha_{\mathrm{ff}}=0.018 Z^{2} g_{\mathrm{ff}} T_{\mathrm{csm}}^{-3 / 2} n_{e} n_{I} \nu^{-2}
$$

where $Z=1$ is the ion charge, $g_{\mathrm{ff}}$ is the gaunt factor at the target frequency, and $n_{e}$ and $n_{I}$ are the electron and ion number densities, which we estimate simply as $n_{e}=n_{I}=\rho_{\mathrm{csm}} / m_{p}$ in this work unless stated otherwise, where $m_{p}$ is the proton mass. The width of the pre-shock CSM is

$$
\Delta r_{\text {ext }}=R_{\text {out }}-R_{f},
$$

and thus $\tau_{\mathrm{ff}}$ is known from Equation 10.

The evolution of $\tau_{\text {ssa }}$ and $\tau_{\mathrm{ff}}$ calculated from the models are shown in Figure 2. In these calculations, we have assumed $\epsilon_{B}=0.1, T_{\mathrm{csm}}=10^{3} \mathrm{~K}, \nu=4.9 \mathrm{GHz}, \mu_{e}=$ $\mu_{I}=1$, and $Z=1$. The sharp elbow on the decline of each curve marks $t_{p}$, the time when the forward shock crosses the outer edge of the CSM. In the next section, we will discuss what drives the normalization. Here we will point out that for most of the time that the shock is in the shell, $\tau_{\text {ssa }}$ and $\tau_{\mathrm{ff}}$ are nearly constant. Independent of $f_{R}$, the models with significant absorption by either process have $\rho_{\text {csm }} \gtrsim 10^{-18} \mathrm{~g} \mathrm{~cm}^{-3}$. Since $\tau_{\text {ssa }}(t)$ and $\mathcal{L}_{\nu \text {,thin }}(t)$ are driven by the synchrotron process, they have similar shapes, with a long tail after the shock has crossed the shell and shells following the same rise independent of $f_{R}$. Contrary to this, the $\tau_{\mathrm{ff}}$ is only important while the shock is in the shell and higher- $f_{R}$ have higher optical depths at all times. In some of the later impact time models, we see that the $\tau_{\text {ff }}$ curves jump to low values at certain time steps. This is a numerical artifact of the shock front identification process and does not affect our later results; for the sake of transparency in our methods and because it does not have a large illustrative impact, we have chosen to not to edit the $\tau_{\mathrm{ff}}(t)$ curves of these models.

\section{PARAMETERIZATION OF OPTICAL DEPTH EVOLUTION}

In this section, we present a parameterization to allow synchrotron self-absorption and free-free (external) absorption optical depths to be reconstructed for an arbitrary CSM shell using Equation 11. An example of the light-curves created with this method can be seen in Figure 3, which shows the light-curve with no absorption (dotted curves), $\tau_{\text {ssa }}$ only (dashed curves), and both sources of absorption (solid curves) for models of two different densities.

The variable for time we will use is

$$
x \equiv t / t_{\mathrm{imp}}
$$

i.e., time is normalized to the time of impact. The time that the forward shock crosses the edge of the CSM shell is the time of peak luminosity in the optically-thin radio light-curves and is denoted by $t_{p}$ in Paper I; therefore, 


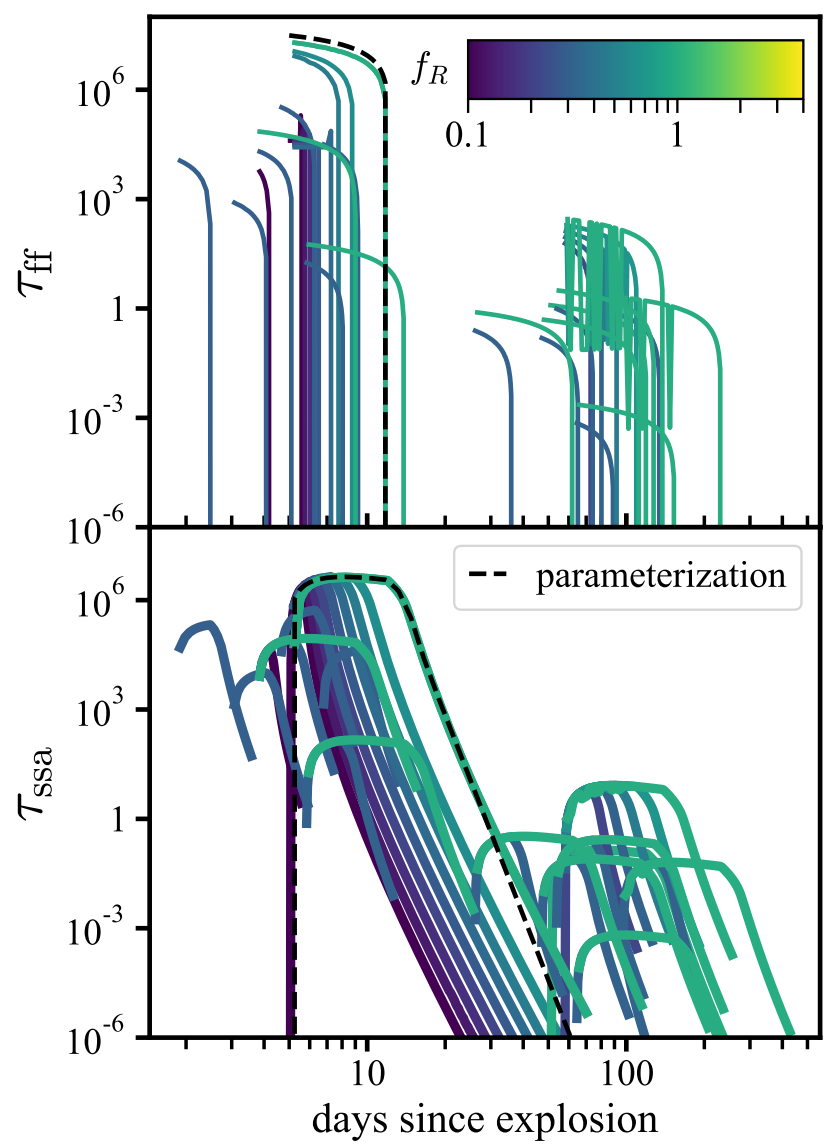

Figure 2. Calculation of $\tau_{\mathrm{ff}}$ (top) and $\tau_{\mathrm{ssa}}$ (bottom) from the models shown in Figure 1, using $\epsilon_{B}=0.1$ (see $\S 4$ ). Density and impact time simply affect the overall position of the evolution (i.e., curves shift up when density increases, and to the right when impact time increases), whereas $f_{R}$ affects the shape of the curve. (Note that the jaggedness of some $\tau_{\text {ff }}(t)$ curves is numerical, not physical) The black dashed curves show an example of applying the parameterization given in $\S 5$ for the shell parameters of our highest optical depth model $\left(\rho_{\mathrm{csm}}=8.8 \times 10^{-16} \mathrm{~g} \mathrm{~cm}^{-3}, f_{R}=1, R_{\text {in }}=\right.$ $\left.9.2 \times 10^{14} \mathrm{~cm}\right)$.

here we will use $x_{p}=t_{p} / t_{\text {imp }}$. As in Paper I, we will provide a functional form for the evolution of $\tau_{\text {ssa }}$ and $\tau_{\text {ff }}$ while $x \leq x_{p}$, and evaluate times that $\tau_{\text {ssa }}$ reaches characteristic values for times $x>x_{p}$ (at which point there is no external absorption because the CSM has been swept over).

\subsection{Synchrotron Self-Absorption}

First we determine the normalization of $\tau_{\text {ssa }}$. Using Equation 52 of Paper I, the normalization of the extinction coefficient is

$$
\alpha_{\mathrm{ssa}} \propto \rho_{\mathrm{csm}}^{-1} u_{\mathrm{gas}}^{13 / 4} \nu^{-7 / 2} \epsilon_{B}^{5 / 4}
$$

From Equation 7 of Paper I, the time evolution of the forward shock radius, $R_{f}$, and shock speed, $v_{s}$, while the shock is in the shell is

$$
\begin{aligned}
x & =0.983\left(R_{f} / R_{c, 0}\right)^{1.28} \\
\Rightarrow R_{f} & =R_{\text {in }} x^{0.781} \\
\text { and } & \\
v_{s} & =\frac{R_{\text {in }}}{t_{\text {imp }}} x^{-0.219}
\end{aligned}
$$

Equation 2 gives $R_{\text {in }}$ in terms of $t_{\text {imp }}$ and $\rho_{\text {csm }}$. Assuming $u_{\text {gas }} \propto \rho_{\text {csm }} v_{s}^{2}$, we now have (dropping factors of $x$ because we are interested in the normalization)

$$
\alpha_{\mathrm{ssa}}(t) \propto \rho_{\mathrm{csm}}^{8 / 5} t_{\mathrm{imp}}^{-1.95} \nu^{-7 / 2} \epsilon_{B}^{5 / 4}
$$

The radial term in $\tau_{\text {ssa }}(\widetilde{\Delta r})$ is the volume-to-area ratio (Equation A6) and should roughly evolve like $R_{f}$ if the shell is thin and has width $\Delta R \propto R_{f}$. Then the expected normalization of $\tau_{\text {ssa }}$ should scale like

$$
\tau_{\mathrm{ssa}} \propto \alpha_{\mathrm{ssa}}(t) R_{f}(t) \propto \rho_{\mathrm{csm}}^{3 / 2} t_{\mathrm{imp}}^{-5 / 4} \nu^{-7 / 2} \epsilon_{B}^{5 / 4}
$$

and we find that, indeed, the evolution of $\tau_{\text {ssa }}(x)$ is the same for all models when normalized by this factor.

Therefore, $\tau_{\text {ssa }}(x)$ at times after impact but before the shock crosses the outer edge of the CSM (i.e., $1 \leq x \leq$ $x_{p}$ ) can be described by the asymptotic function

$$
\begin{aligned}
\tau_{\mathrm{ssa}}(x) & =13.6\left(\frac{\rho_{\mathrm{csm}}}{10^{-18} \mathrm{~g} \mathrm{~cm}^{-3}}\right)^{3 / 2}\left(\frac{t_{\mathrm{imp}}}{100 \text { days }}\right)^{-5 / 4} \\
& \times\left(\frac{\nu}{4.9 \mathrm{GHz}}\right)^{-7 / 2}\left(\frac{\epsilon_{B}}{0.1}\right)^{5 / 4} \\
& \times x^{-1.34}\left(1-x^{-1.66}\right)
\end{aligned}
$$

The normalization factor and the exponents in the asymptotic function $x^{-1.34}\left(1-x^{-1.66}\right)$ were determined using scipy.optimize. curve_fit.

As with the optically thin luminosity, we fit the evolution of $\tau_{\text {ssa }}$ after the shock has crossed the outer edge of the CSM (i.e., $x>x_{p}$ ) by determining the time at which $\tau_{\text {ssa }}$ reaches characteristic fractions of $\tau_{\text {ssa }, p} \equiv \tau_{\text {ssa }}\left(x_{p}\right)$ and connecting the points with power-laws (i.e., linear interpolation in logarithmic space). The characteristic points are

$$
\begin{gathered}
x\left(\tau_{\mathrm{ssa}}=0.5 \tau_{\mathrm{ssa}, p}\right)=1.015\left(1+f_{R}\right)^{1.38} \\
x\left(\tau_{\mathrm{ssa}}=0.1 \tau_{\mathrm{ssa}, p}\right)=1.046\left(1+f_{R}\right)^{1.49} \\
x\left(\tau_{\mathrm{ssa}}=10^{-2} \tau_{\mathrm{ssa}, p}\right)=1.118\left(1+f_{R}\right)^{1.54} \\
x\left(\tau_{\mathrm{ssa}}=10^{-3} \tau_{\mathrm{ssa}, p}\right)=1.206\left(1+f_{R}\right)^{1.60} .
\end{gathered}
$$


Beyond this latest time point we assume adiabatic evolution,

$$
\begin{aligned}
& \alpha_{\mathrm{ssa}} \\
& \propto t^{-3}\left(t^{-5}\right)^{13 / 4} \propto t^{-19.25} \\
\Rightarrow r & \propto t \\
\Rightarrow & \tau_{\mathrm{ssa}} \propto t^{-18.25} .
\end{aligned}
$$

where we have assumed the shell inner and outer radii evolve like $r \propto t$, density evolves like $\rho \propto t^{-3}$, and energy density evolves like $u \propto V^{-5 / 3} \propto t^{-5}$, with $V$ being the shell volume.

\subsection{Free-Free Absorption}

For the external, free-free absorption, the evolution of optical depth reflects the radial evolution of the shock, i.e.,

$$
\tau_{\mathrm{ff}}(x)=\alpha_{\mathrm{ff}} \Delta r_{\mathrm{ext}}(x) .
$$

Using Equation 5, this can be estimated as

$$
\Delta r_{\mathrm{ext}}(x)=R_{\mathrm{in}}\left[\left(1+f_{R}\right)-x^{0.781}\right],
$$

where we have used $0.987 \approx 1$ to make it exact at $x=1$ rather than using the fit value, so,

$$
\tau_{\mathrm{ff}}(x)=\alpha_{\mathrm{ff}} R_{\mathrm{in}}\left[\left(1+f_{R}\right)-x^{0.781}\right],
$$

with $\alpha_{\mathrm{ff}}$ as in Equation 15 and $R_{\text {in }}$ given by Equation 2 .

Note that $\tau_{\mathrm{ff}}\left(x>x_{p}\right)=0$ because $x_{p}$ represents the time at which the forward shock crosses the edge of the CSM shell, thus, all of the CSM has been shocked and there is no "external" medium.

\subsection{Error of the Parameterization}

The error incurred by using the fitting functions given above - i.e., comparing $\tau_{\mathrm{ssa}}(x)$ and $\tau_{\mathrm{ff}}(x)$ calculated with the given formulae versus from the simulations themselves - is small, $\lesssim 30 \%$ on each, near the peak of the optically thin light-curve. Very near the time of impact, when the system is changing rapidly, the error can be much larger. We also find that the adiabatic approximation does not match the very late time behavior well, possibly due to deceleration from the "interstellar medium" gas (of density $10^{-24} \mathrm{~g} \mathrm{~cm}^{-3}$ ) that lies outside the shells. It is unlikely that either of these phases will be of practical use to the interpretation of observations, since the (optically thin) luminosity of the shocked gas is so low at these times $-\lesssim 0.1 \%$ of the optically thin peak luminosity. Nevertheless, we caution that one take care if interpretation of observed data hinges on the very early or late phases of the interaction.

\section{APPLICATION TO RADIO DATASETS}

In this section we show how the parameterized lightcurves can be applied to radio datasets. For these analyses, we assume $T_{\mathrm{csm}}=10^{4} \mathrm{~K}$ when calculating the freefree absorption, i.e., that the preshock CSM is heated similar to an HII region by the ionizing radiation of the shock.

\subsection{Testing Models of PTF11kx}

Dilday et al. (2012) report a non-detection of PTF11kx with the Karl G. Jansky Very Large Array (VLA) obtained on March 30, 2011 with a $1 \sigma$ rootmean-square image noise of $23 \mu \mathrm{Jy}$. This is +61 days since $B$-band maximum (January 29, 2011). From the NRAO archive, we find that the central frequency of the observation was $8.4 \mathrm{GHz}$.

Consistent with Graham et al. (2017), in this analysis, we assume a distance of $204.4 \mathrm{Mpc}$ and that $B$ band maximum occurs 13 days after explosion, interaction began at $t_{\mathrm{imp}}=50$ days, and interaction ended at $t_{p}=500$ days. Variations in these timings of $\sim 10 \%$ do not affect our conclusions. Within our model framework, we can derive $f_{R}$ from $t_{p}$ and $t_{\text {imp }}$ (Equation 4). The only other necessary model input is the density of the shell $\rho_{\text {csm }}$, which can be combined with $t_{\text {imp }}$ to find $R_{\text {in }}$.

For PTF11kx, $\rho_{\text {csm }}$ can be estimated from its optical spectra. The Ca II H\&K absorption lines were saturated at early times, allowing an inference of the CSM column density, $N_{\text {csm }}$, assuming solar composition. The mass density, $\rho_{\text {csm }}$, can be found from $N_{\text {csm }}$ if we assume the density is constant within the shell and if the extent of the CSM $\left(\Delta R=f_{R} R_{\text {in }}\right)$ and mean particle weight $(\bar{m})$ are known, as $\rho_{\mathrm{csm}}=\bar{m} N_{\mathrm{csm}} / \Delta R$. We take $\bar{m}$ is 1.33 times the proton mass, as appropriate for neutral material of solar abundance. Graham et al. (2017) derive $N_{\mathrm{csm}} \approx 5 \times 10^{21} \mathrm{~cm}^{-2}$, significantly lower than the original estimate by Dilday et al. (2012) of $N_{\text {csm }} \approx 10^{23} \mathrm{~cm}^{-2}$. The lower estimate is probably correct, for two reasons. First, because two different methods for analyzing the line indicate a lower $N_{\mathrm{csm}}$ (Graham et al. 2017). Second, the higher value of $N_{\text {csm }}$ creates an inconsistency - to create a saturated line requires that the CSM cover the SN photosphere, but full coverage implies a high CSM mass that is inconsistent with the weak levels of interaction seen (Dilday et al. 2012). Although we favor the lower density estimate, we will investigate both hypotheses.

In Figure 3 we compare the radio limit for PTF11kx to the radio light-curves based on the current best descriptions of its CSM as described above. In calculating $\tau_{\mathrm{ff}}$, we have assumed $\mu_{I}=1.33, \mu_{e}=1.18$ to be 


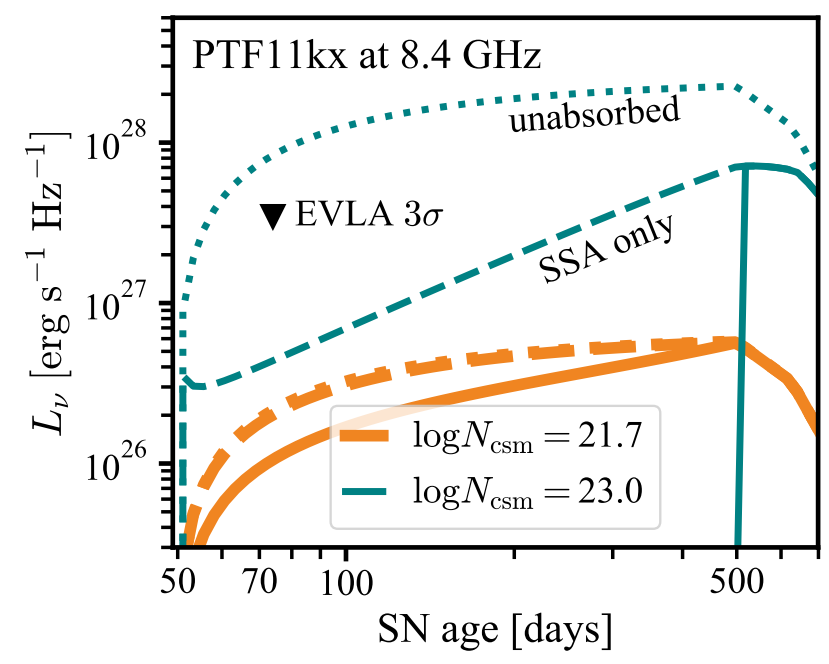

Figure 3. The VLA $3 \sigma$ radio limit for PTF11kx (black triangle) is consistent with models for either $N_{\mathrm{csm}} \approx$ $10^{23} \mathrm{~cm}^{-2}$ (blue) or $N_{\mathrm{csm}} \approx 5 \times 10^{21} \mathrm{~cm}^{-2}$ (orange). Dotted curves show the optically thin light-curves, dashed curves include synchrotron self-absorption $\left(\epsilon_{B}=0.1\right)$, and solid curves furthermore include free-free absorption (with $\left.Z=1, \mu_{e}=1.18, \mu_{I}=1.33, T_{\mathrm{csm}}=10^{4} \mathrm{~K}\right)$. A radio observation at $\sim 500$ days may have distinguished between the $N_{\text {csm }}$ measurements.

consistent with the compositional assumptions used for determining $N_{\text {csm }}$. We find that with either estimate of $N_{\mathrm{csm}}$, our models are consistent with the radio nondetection of PTF11kx. Our optically thick light-curves are needed to interpret the high- $N_{\text {csm }}$ scenario, whereas the low- $N_{\text {csm }}$ case is subject to very little absorption. If a second observation had been taken around one year after explosion, it would have been able to distinguish between the $N_{\text {csm }}$ values. That is, under the assumption of spherically distributed CSM. When one allows the CSM to be in a torus, the light-curves must be modified; this should roughly be a diminution of the luminosity by the covering fraction of the CSM, if we saw PTF11kx edgeon as suggested by the saturated pre-impact absorption lines, which would make the signal too dim to be seen by the VLA observation.

\subsection{The Allowed Fraction of SNe Ia with CSM Shells}

Using the optically thick light-curve parameterization (§3) we can explore the detection power of radio upperlimits for CSM shells. While a similar analysis has been carried out for individual objects (Harris et al. 2018; Cendes et al. 2020; Pellegrino et al. 2020), this is the first such analysis of a population of SNe Ia. In this analysis we assume $\mu_{e}=\mu_{I}=1, Z=1$, and $T_{\mathrm{csm}}=10^{4} \mathrm{~K}$.

Chomiuk et al. (2016) present VLA observations of thermonuclear supernovae (SNe Ia) across all sub-groups

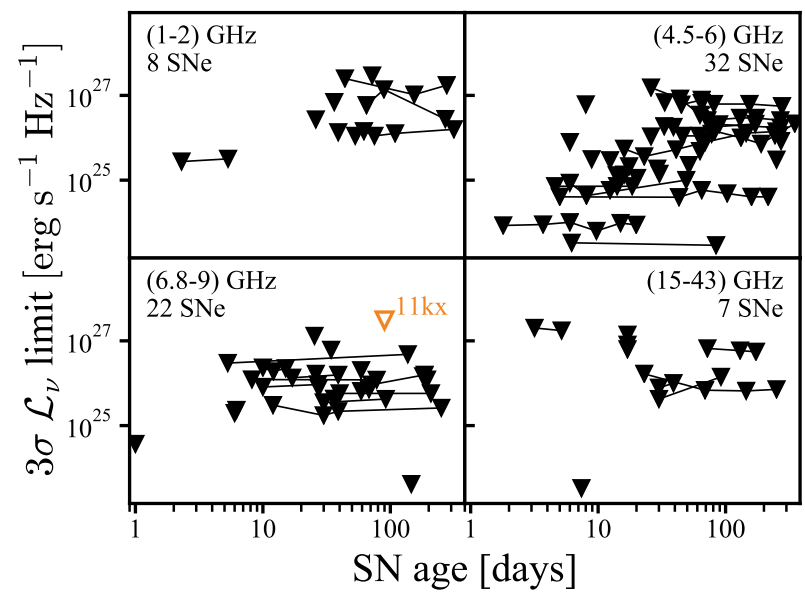

Figure 4. Data ( $3 \sigma$ upper limits) used to determine the fraction of SNe Ia that may host shells.

of the class. Of these, we use the "cool," "shallowsilicon," and "core-normal" groups. We also incorporate data compiled in Lundqvist et al. (2020) and those presented in Mooley et al. (2016) and Ryder et al. (2019). We group the three sub-types together since the cool and shallow-silicon groups are too sparsely sampled to be analyzed independently. The sample of data, shown in Figure 4, covers a range of frequencies from 1-43 GHz. Observations span $1-365$ days after explosion for a total of 50 SNe among all observations independent of frequency (observations are grouped by frequency here for comparison with Figure 5). We show PTF11kx in this figure for reference; this data point is not included in our following statistical analysis because it is not a useful limit, i.e., it would not be able to detect any model in our set of interest.

In this analysis, we characterize CSM shells by their mass $\left(M_{\mathrm{csm}}\right)$, inner radius $\left(R_{\mathrm{in}}\right)$, and fractional width $\left(f_{R} \equiv \Delta R / R_{\text {in }}\right)$. These three parameters fully determine a shell light-curve in our model framework. We are interested in constraining the fraction of SNe Ia with CSM of a given $M_{\text {csm }}$ and $f_{R}$, which we will call the CSM's "configuration."

We must choose a distribution of $R_{\text {in }}$ to make this constraint, which we do as follows. Note that we will use $R_{\text {in }, 16}=R_{\text {in }} /\left(10^{16} \mathrm{~cm}\right)$. Moore \& Bildsten (2012) used analytic calculations to explore the CSM established by recurrent nova eruptions in a binary system with a red giant companion and significant associated winds. They found that the nova ejecta sweep up the giant wind and quickly (within 20 years) and decelerate to a drastically reduced coasting speed of $\lesssim 100 \mathrm{~km} \mathrm{~s}^{-1}$. The exact values depend on the recurrence time and companion wind mass-loss rate. Due to the low speed, the shells build up into a thicker, more massive shell than would be formed 
from an individual nova eruption. This slow shell is formed at a distance $R_{\mathrm{in}, 16} \sim 0.1-10$, depending on the binary parameters. Traveling at $\lesssim 100 \mathrm{~km} \mathrm{~s}^{-1}$, the thick shell will remain in the system for $10^{4}-10^{5}$ yr before mixing into the interstellar medium - giving plenty of time for a massive shell to build up if the recurrent novae continue.

We consider the delay time between shell formation and SN explosion to be entirely unknown (i.e., that the $\mathrm{SN}$ event is equally likely to occur at any time after the start of the recurrent nova period begins). Therefore, the probability distribution for $R_{\text {in }}$ is determined by the shell kinematics. Since the shells spend only 20 years within $r \sim 10^{15} \mathrm{~cm}$ compared to the $>10^{4}$ years they spend beyond this distance, we treat the probability of $R_{\text {in, } 16}<0.1$ as zero. Because a shell coasts at constant speed, all radii $R_{\mathrm{in}, 16} \geq 0.1$ are equally likely. We only analyze the probability of SNe Ia having shells with $R_{\mathrm{in}, 16} \in[0.1,1]$, i.e., the range that can be studied through observations within a year of explosion (the data; Chomiuk et al. 2016 specifically limited their survey to radio observations obtained in the first year following explosion). Note that because the shells are freely expanding, $t_{\text {imp }} \propto R_{\text {in }}$ and $f_{R}(=\Delta R / R \propto t / t)$ is constant as it moves away from the binary.

The radio light-curves for the shell configurations we consider are summarized in Figure 5.

In the top panel, we show all light-curves generated for just one shell configuration in different frequency bins. In the actual analysis, a light-curve is generated at the the frequency of each individual observation. To determine if an observation has constraining power in a situation where the location of the CSM is unknown, one must not compare a luminosity limit to a single lightcurve but instead look at the light-curve "roof" that is created by the set of possibilities (black dashed line). Observations under the roof have constraining power, whereas anything above the roof has no possibility of detecting any shell and therefore no statistical power.

In the bottom panel, we show the roofs for other shell configurations, spanning $M_{\mathrm{csm}}=10^{-4}-0.1 M_{\odot}$ and $f_{R}=0.1-1$. The dark green curve (farthest right) is the same as the black dotted line from the top panel at $5 \mathrm{GHz}$, the frequency with the most $\mathrm{SNe}$ observed. Observations in the shaded regions (i.e., under the roof) have constraining power on the configuration. The black dashed line shows the typical luminosity limit of the data, which is very close to the top of the "roof" for most models, and has best coverage for the higher-mass $f_{R}=1$ shells - i.e., these are the shells most suited for study by the radio observations. Again we note that the PTF11kx observation shown in Figures 3 and 4 is above
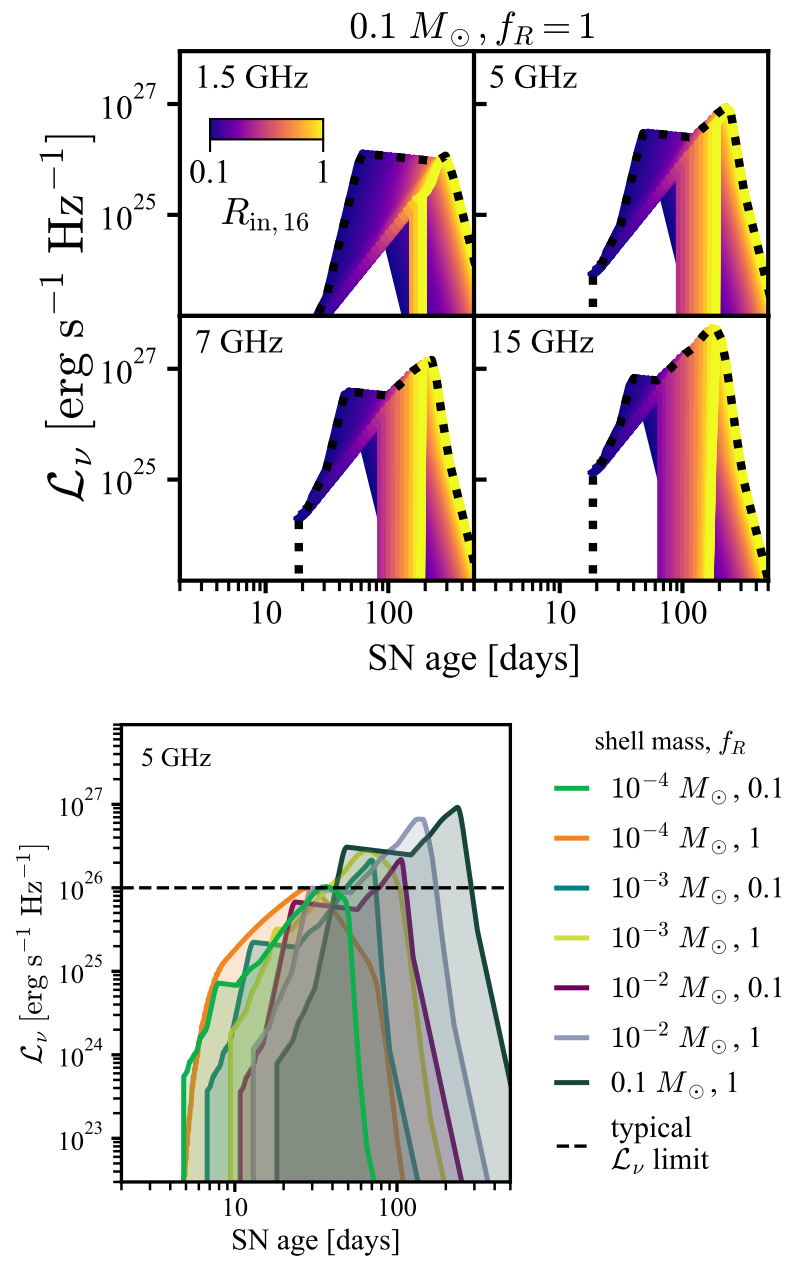

Figure 5. Top: A grid of light-curves for a single shell configuration $\left(M_{\mathrm{csm}}=0.1 M_{\odot}, f_{R}=1\right)$, where $R_{\mathrm{in}, 16}$ varies between $0.1-1$ (denoted by color scale ranging from magenta to yellow). The light-curve grid forms a "roof" at a given frequency (black dotted line); observations looking to sample this shell configuration must be under the roof. Bottom: $5 \mathrm{GHz}$ fiducial light-curves showing roofs for various shell configurations $\left(R_{\mathrm{in}, 16} \in[0.1,1]\right)$. Color represents the shell configuration (see legend). The black dashed line shows the median $3 \sigma$ luminosity limit of the SN Ia sample (Figure 4), and the $\mathrm{x}$-axis range has been chosen to span the observation times. Observations are primarily under the roofs of $f_{R}=1$ models, so radio observations will be most constraining for these shells.

the roofs, so it is not useful to our analysis. Note that CSM parameters that would violate model assumptions (as described in $\S 2$ ) are not shown, which is one reason that the low-mass shell roofs look different from those of higher masses.

The probability of detecting interaction with a CSM shell of mass $M_{\mathrm{csm}}$ and fractional width $f_{R}$ in an SN Ia event is the product of (a) the fraction of SNe Ia that host such shells $(\xi)$ and (b) the probability that ob- 
servations of the SN can detect the interaction signal $\left(\mathcal{P}_{i}(\operatorname{det} \mid\right.$ occ $\left.)\right)$. The former term is the one of interest to our study, and it can range from $\xi \in[0,1]$.

The latter term we calculate by creating a grid of model light-curves with $R_{\text {in,16 }} \in[0.1,1]$, then injecting these light-curves into the set of $3 \sigma$ upper-limits for the observed SN sample (i.e., Figure 4). The number of injected light-curves that would be detectable for $\mathrm{SN} i\left(N_{\mathrm{det}, i}\right)$ compared to the number in the grid $(N)$ is a good estimation of the detection probability for the shell, provided that the number of models is high enough:

$$
\mathcal{P}_{i}(\operatorname{det} \mid \text { occ })=\frac{N_{\text {det }, i}}{N}
$$

We use $N=100$ models. Thus for each SN the probability of detecting interaction is

$$
\mathcal{P}_{i}(\operatorname{det})=\xi \frac{N_{\mathrm{det}, i}}{N} .
$$

The probability that of $S$ events, none discovered interaction with a shell is

$$
\mathcal{P}(\text { no dets })=\prod_{i=0}^{S}\left(1-\xi \frac{N_{\mathrm{det}, i}}{N}\right) .
$$

In a Bayesian framework, the probability density $p$ of a given value of $\xi$ being true is

$$
p(\xi \mid \text { no } \operatorname{dets}, I) \propto p(\text { no } \operatorname{dets} \mid \xi, I) \times p(\xi \mid I),
$$

where $I$ represents our model assumptions. Since our model assumptions do not depend on the fraction of SNe Ia with CSM shells, $p(\xi \mid I)=1$. The probability density $p$ (no $\operatorname{dets} \mid \xi, I)$ is proportional to $\mathcal{P}$ (no dets).

Thus the observed non-detections can be transformed into an upper limit on $\xi$ via

$$
\mathcal{P}\left(\xi<\xi_{\text {up }}\right)=\frac{\int_{0}^{\xi_{\text {up }}} \prod_{i=0}^{S}\left(1-\xi^{\prime} \frac{N_{\mathrm{det}, i}}{N}\right) d \xi^{\prime}}{\int_{0}^{1} \prod_{i=0}^{S}\left(1-\xi^{\prime} \frac{N_{\mathrm{det}, i}}{N}\right) d \xi^{\prime}}
$$

and we can obtain the maximum allowed value of $\xi$ at $99.7 \%$-confidence $(3 \sigma)$ by finding the $\xi_{\text {up }}$ at which Equation 41 evaluates to 0.997 .

Figure 6 shows the results of the analysis, providing $99.7 \%$ confidence limits on $\xi$, assuming $\epsilon_{B}=0.1$ (large markers, solid lines) and $\epsilon_{B}=0.01$ (small markers, dotted lines). We see that in all cases, the large sample of radio non-detections is still consistent with a high fraction of SNe Ia having confined CSM shells - especially if $\epsilon_{B}=0.01$ is the appropriate value for these shocks.

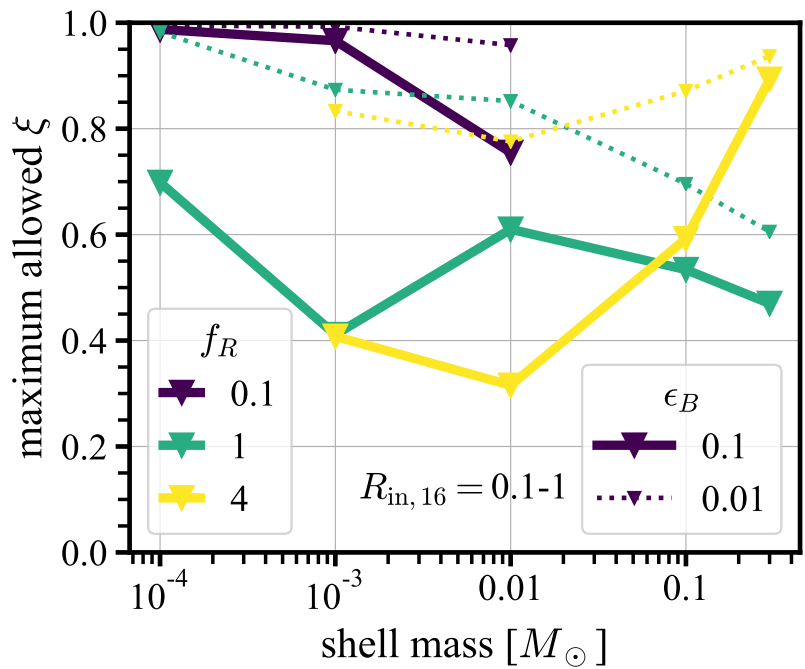

Figure 6. Maximum fraction of $\mathrm{SNe} \mathrm{Ia}(\xi)$ that can have CSM shells of a given mass and width, within $R_{\text {in }}=10^{16} \mathrm{~cm}$. The value of $\xi$ is calculated at $3 \sigma(99.7 \%)$ confidence. Large markers are calculations with $\epsilon_{B}=0.1$, while small markers represent $\epsilon_{B}=0.01$. The shell with $M_{\mathrm{csm}}=0.3 M_{\odot}$ and $f_{R}=4$ represents a PTF $11 \mathrm{kx}$-like configuration.

Single nova outbursts. - The thickness of a single nova outburst is predicted to be $f_{R} \sim 0.1$ in Moore \& Bildsten (2012); these should also be low mass (e.g., $\sim 10^{-4} M_{\odot}$ Chomiuk et al. 2014). We see that nova-like shells (thin and low-mass) are currently largely unconstrained by radio observations. Essentially all $\mathrm{SNe}$ Ia could have a $10^{-4} M_{\odot}, f_{R}=0.1$ shell hiding in their circumstellar environment, according to these radio data as interpreted in our model framework. Nova ejecta spread over a larger volume $\left(f_{R}=1\right)$ are only constrained to $\lesssim 70 \%$ of all SNe Ia, assuming $\epsilon_{B}=0.1$.

Multiple novae. - Multiple nova eruptions could produce a thicker, more massive shell. We see that (for $\epsilon_{B}=$ $0.1), f_{R}=1$ shells are constrained to be $\lesssim 50 \%$ across the mass range explored. This is because, as can be seen in Figure 5, these models have similar requirements for their observability - higher mass shells are more luminous (in the optically thin limit) but are also subject to more absorption.

Very thick (PTF11kx-like) shells. - In our analysis, we include a $0.3 M_{\odot}$ shell with $f_{R}=4$, representing a PTF11kx-like CSM (Graham et al. 2017). From the radio limits alone, we find that up to $90 \%$ of SNe Ia could have CSM with a PTF11kx-like configuration. Lower mass, thick shells are more constrained because they have a lower free-free optical depth, yet we see that the radio non-detections are still consistent with a relatively high fraction of SNe Ia having thick shells. 


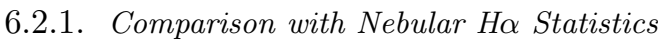

Recently, Tucker et al. (2020) used a sample of 111 low-redshift SNe Ia to constrain the dominance of the single-degenerate channel in creating $\mathrm{SNe}$ Ia using the theoretical framework of Botyánszki et al. (2018). These models focus on the $\mathrm{H} \alpha$ signature from hydrogen that has been stripped off the companion envelope, and they allow one to convert flux limits into limits on the mass of stripped material (subject, of course, to a variety of underlying model assumptions), which can then be compared to theoretical expectations.

One striking decision made in the Tucker et al. (2020) analysis was to exclude all known cases of SNe Ia with late-time $\mathrm{H} \alpha$ emission, even if those events looked normal near maximum light and had observations in the same time frame as the rest of the sample - the 91T-like ("shallow silicon") PTF 11kx and the 91bg-like ("cool") events SN 2018fhw and SN 2018cqj (Dilday et al. 2012; Kollmeier et al. 2019; Prieto et al. 2020). Another example of a 91T-like SN Ia with late-time $\mathrm{H} \alpha$ emission, but that unfortunately does not have observations in the time window considered, is SN 2015cp (Graham et al. 2019). Tucker et al. (2020) essentially argue that these events ought to be excluded because they are abnormal; but, by definition, hydrogen emission is abnormal in any SN I. We note that there is a strong distinction between the 91T-like delayed-interaction events and 91bg-like cases; pertinent to this discussion, 91bg-like cases have low-luminosity line emission and may represent stripped companion material, whereas 91T-like cases have higher line luminosity and a distinct CSM origin. Therefore, it was sensible for Tucker et al. (2020) not to analyze PTF11kx or SN 2015cp in the stripped-companion model framework, since the line signal was not of this origin. However, we note that the $91 \mathrm{bg}$-like events had estimated stripped masses of $\sim 10^{-2}-10^{-3} M_{\odot}$, and we estimate that the Tucker et al. (2020) have 65 events that probe a similar mass (their Figure 6), therefore had these events been included in the nebular sample the statistics would have been two detections among 67 events, which under a simple binomial distribution analysis results in a $3 \sigma$ limit of $(0.3-13.4) \%$ of SNe Ia with $\sim 10^{-2}-10^{-3} M_{\odot}$ of (stripped) hydrogen.

Our study, however, is not concerned with the signature of stripped material, but rather with circumstellar material. We do not yet have $\mathrm{H} \alpha$ emission models for the CSM shell scenario investigated in this work, so we cannot perform an analysis we have done for the radio using the Tucker et al. (2020) data. However, what we can say is that any of the Tucker et al. (2020) observations would have been able to detect $\mathrm{H} \alpha$ emission from a PTF11kx twin. Including the other three events into the statistics is complicated by their lower luminosity (SN 2018cqj), observations being earlier than the rest of the sample (SN 2018fhw), or observations being later than the rest of the sample (SN 2015cp). If Tucker et al. (2020) had chosen to include PTF11kx in their sample, then they would have 104 normal, 91T-like, or 91bg-like events in their sample (the categories we analyze in this study) and one detection of $\mathrm{H} \alpha$ emission in the 3-15 months after maximum light time window, resulting in an allowed fraction of of SNe Ia with CSM like PTF11kx of $(0.03-7.21) \%$ at $99.7 \%$ confidence. In comparison, our limit from radio data is that up to $\sim 90 \%$ of SNe Ia could have a PTF11kx-like shell (because, for the majority of the interaction, we predict the radio emission is absorbed by the preshock CSM).

One point of interest to both the radio and optical studies of delayed interaction is that hydrogen emission in SNe Ia - regardless of its time of appearance is so far associated with 91bg-like or 91T-like SNe Ia (see the above references for individual events with latetime hydrogen emission as well as Leloudas et al. 2015), which are relatively rare. Therefore, even large samples, like those discussed in this work, will not provide a statistically significant number of events from these subgroups. For example, if PTF11kx had been included in Tucker et al. (2020), the sample size of 91T-like events would be six, with one detection, and the prevalence of PTF 11kx-like objects constrained to $(0.8-77.1) \%$ of 91T-like SNe Ia - or (0.6-79.9)\%, if SN $2015 \mathrm{cp}$ is included also. For $91 \mathrm{bg}$-like events, the sample would become ten events with two detections, and the fractional limit constrained to (0.9-75.6)\% of 91bg-like events similar to SN 2018fhw. If these subgroups represent the single-degenerate channel, as has been suggested on theoretical grounds by Fisher \& Jumper (2015), then these statistics highlight how little we know about SNe Ia that do come from the single-degenerate channel compared to the constraints that have been made on the prevalence of the single-degenerate channel overall. Furthermore, no SN Ia with hydrogen emission fits neatly into the singledegenerate progenitor picture (having either too much or too little hydrogen mass inferred, and nothing that looks like a normal stellar wind) which challenges our picture of this pathway to explosion - and therefore, challenges some of the very models used to constrain its prevalence among SNe Ia.

\section{SUMMARY}

SNe Ia with detached, confined shells of CSM (which produce $\mathrm{SNe} I \mathrm{I} ; \mathrm{n})$ provide a window into the singledegenerate channel and may represent SNe Ia impacting a CSM shaped by novae. However, the uncertain 
mass, extent, and location of these shells makes it challenging to observe them in an interacting phase, creating large uncertainty in the intrinsic prevalence of these shells. Adding to this uncertainty, and what this work aims to alleviate, is the need for theoretical tools that can interpret SN Ia observations in the context of interaction with these shells - because observations are taken during periods of hydrodynamic transition, popular equations based on asymptotic solutions cannot be accurately applied and new ones must be found. Without appropriate modeling, the properties of these shells cannot be precisely determined (limiting studies of their origin), nor can their occurrence rate be assessed from an SN Ia survey.

In Harris et al. (2016, Paper I, summarized in § 2), we presented hydrodynamic models of shell interaction scenarios for thin, low mass shells and their corresponding optically thin synchrotron radio light-curves. We found a parameterization to reproduce the light-curve of a shell interaction given the shell properties. In this paper, we have extended those results to account for synchrotron self-absorption and free-free absorption. This allows an exploration of higher-density shells than was possible from the results of that work.

In $\S 3$ we describe our method for using the optical depth to synchrotron self-absorption $\left(\tau_{\text {ssa }}\right)$ and free-free absorption $\left(\tau_{\mathrm{ff}}\right)$ to obtain the escape fraction of the radio photons. We find that around a shell density of $\rho_{\mathrm{csm}} \gtrsim 10^{-18} \mathrm{~g} \mathrm{~cm}^{-3}$, both sources of absorption begin to come into play. We then derive $4.9 \mathrm{GHz} \tau_{\text {ssa }}$ and $\tau_{\text {ff }}$ values from the hydrodynamic model suite, which requires finding the shock width and mean extinction coefficient as a function of time $(\S 4)$, to explore how these quantities evolve over time. In $\S 5$ we showed how the optical depth evolution calculated from the simulations can be parameterized in a similar way to the optically thin light-curves, allowing for these quantities to be calculated once the shell properties are specified. For convenience of use, these parameterizations are implemented in a Python script (HNK16_tools.py) available online. $^{2}$

In $\S 6$ we apply this new tool to radio observations of SNe Ia. First, we consider the radio non-detection of PTF11kx (Dilday et al. 2012) and assess whether it is consistent with the current picture of its CSM (Silverman et al. 2013; Graham et al. 2017) - a $\sim 0.3 M_{\odot}$ shell extending from $\sim 10^{16} \mathrm{~cm}$ to $\sim 5 \times 10^{16} \mathrm{~cm}$. We find that the radio non-detection is consistent with this model, and the non-detection limit was well above the

\footnotetext{
2 https://github.com/chelseaharris/csmpy
}

maximum radio luminosity reached by the interaction at any phase. Dilday et al. (2012) originally proposed a higher density of CSM, and we show that (if this had been spherically distributed) it would have reached a detectable level, but only at late times ( $\sim 1$ year postexplosion), when free-free absorption no longer played a role, which may be a worthy consideration for future radio studies of SNe Ia with stronger interaction.

Second, we use the optically thick light-curve models to statistically assess an ensemble of $\mathrm{SN}$ Ia radio non-detections at various times and frequencies. The parameter of interest is $\xi$, the fraction of SNe Ia that host a shell of mass $M_{\mathrm{csm}}$ and fractional width $f_{R}$ at a distance of $10^{15}-10^{16} \mathrm{~cm}$. We consider $M_{\mathrm{csm}}$ between $10^{-4} M_{\odot}$ and $0.3 M_{\odot}$ with $f_{R}=0.1,1$, and 4 . Overall, we find that, at $99.97 \%$ statistical confidence, thick shells $\left(f_{R}=1,4\right)$ of any mass $<0.1 M_{\odot}$ can be present in up to $\xi \sim 60 \%$ of SNe Ia and still be consistent with the radio non-detections. Thin shells are essentially completely unconstrained. Surprisingly, PTF11kx-like shells, which should be relatively easy to see in optical spectra, are only constrained by radio data to be in $\lesssim 90 \%$ of SN Ia systems, because these relatively massive and thick shells are more subject to free-free absorption. We further calculate the constraints under the assumption of weaker magnetic field amplification $\epsilon_{B}=0.01$, in which case the radio limits allow a large majority of SNe Ia to host shells.

\section{ACKNOWLEDGMENTS}

C.E.H. and L.C. are grateful for support from NSF through AST-1751874 and AST-1907790. C.E.H. also acknowledges support from the Packard Foundation.P.E.N. acknowledges support from the DOE under grant DE-AC02-05CH11231, Analytical Modeling for Extreme-Scale Computing Environments.

We thank the anonymous reviewer for their comments on this manuscript.

This research used resources of the National Energy Research Scientific Computing Center, a DOE Office of Science User Facility supported by the Office of Science of the U.S. Department of Energy under Contract No. DE-AC02-05CH11231.

The National Radio Astronomy Observatory is a facility of the National Science Foundation operated under cooperative agreement by Associated Universities, Inc.

Michigan State University occupies the ancestral, traditional, and contemporary Lands of the Anishinaabeg-Three Fires Confederacy of Ojibwe, Odawa, and 


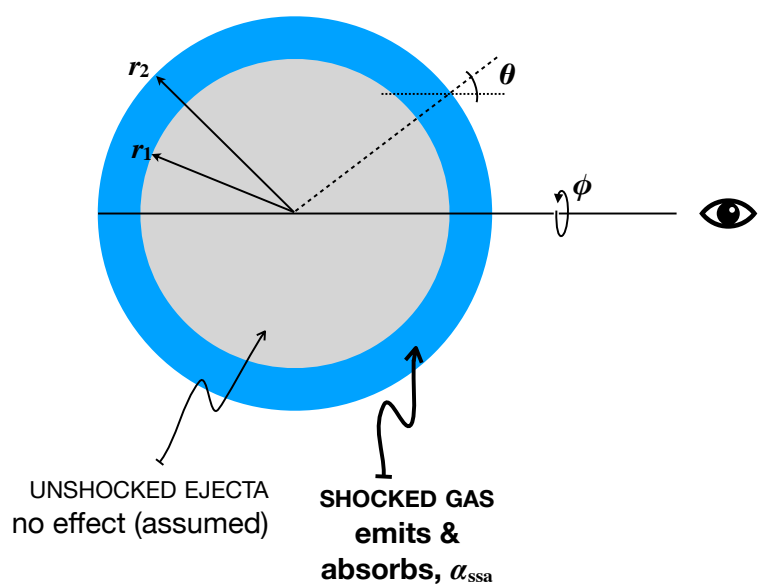

External Absorbing Medium

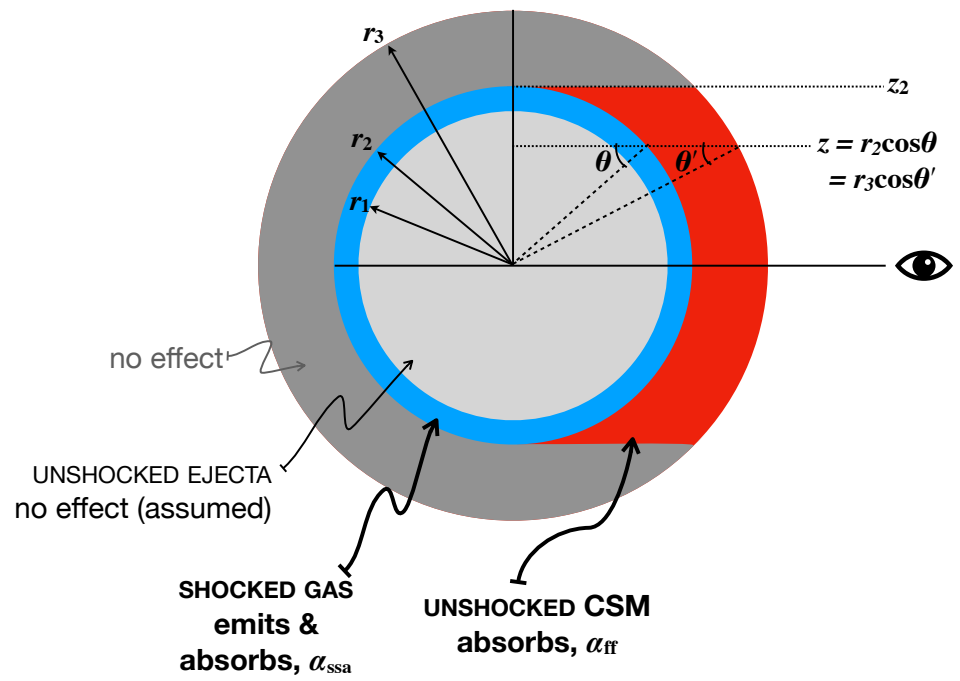

Figure 7. Schematic of the geometries considered for solving the radiation transport equation: the case with no external absorption (left) and with external absorption (right). The ejecta absorption is assumed to have no effect because the contribution of the obscured emission region to the overall luminosity is small.

Potawatomi peoples. The University resides on Land ceded in the 1819 Treaty of Saginaw.

Facilities: Karl G. Jansky Very Large Array
Software: Sedona (Kasen et al. 2006), SciPy (Jones et al. 2001), NumpPy (Oliphant 2006), Astropy (Astropy Collaboration et al. 2013), Matplotlib (Hunter 2007)

\section{APPENDIX}

\section{A. RAY TRACING IN A SPHERICAL SHELL GEOMETRY}

In the optically thin limit of isotropic emission, the spectral (or "specific") luminosity of an emitting shell can be simply calculated as

$$
\mathcal{L}_{\nu, \text { thin }}=4 \pi j_{\nu} V
$$

where $j_{\nu}$ is the emissivity (units like $\mathrm{erg} \mathrm{s}^{-1} \mathrm{~Hz}^{-1} \mathrm{~cm}^{-3} \mathrm{str}^{-1}$, value is assumed constant throughout the shell), $V$ is the volume (units like $\mathrm{cm}^{3}$ ), and the factor of $4 \pi$ accounts for the angle covered by the emission (thus has units of str [sterradians]). In the case that the shell both emits and absorbs light, however, one must solve the radiation transport equation.

\section{A.1. Internal Absorption Only}

Consider a thin, spherical shell extending from radius $r_{1}$ to $r_{2}$, with constant extinction coefficient ( $\alpha$, units like $\mathrm{cm}^{-1}$ ) and $j_{\nu}$ within the shell and no emission or absorption in the cavity $r<r_{1}$. This is the scenario if one assumes (1) absorption by the unshocked ejecta is negligible and (2) absorption by the unshocked CSM is negligible, either due to the CSM column density or because the shock has already crossed the outer edge of the CSM shell. (Note that even if the ejecta full absorb the radio emission, it will have a negligible effect on the overall radio luminosity because most of the emission comes from the projected inner edge of the radiating shell, as is familiar from spatially-resolved examples of interaction, such as the $\mathrm{H} \alpha$ emission of SN remnants or radio interferometry of interacting SNe.)

Then the solution to the radiation transport equation along a straight path through the sphere that makes an angle $\theta$ with the outward surface normal at $r_{2}$ is

$$
I_{\nu}(\theta)=\frac{j_{\nu}}{\alpha}\{1-\exp [-\tau(\theta)]\}=\frac{L_{\nu, \text { thin }}}{4 \pi V \alpha}\{1-\exp [-\tau(\theta)]\},
$$


where $I_{\nu}$ is the specific intensity (units like $\mathrm{erg} \mathrm{s}^{-1} \mathrm{~Hz}^{-1} \mathrm{~cm}^{-2} \mathrm{str}^{-1}$ ), $\tau$ is the optical depth ( $\alpha$ multipied by the path length), and in the second expression we have substituted in the equation for optically thin luminosity. Defining $\tau_{2} \equiv \alpha r_{2}$ and $\theta_{1}$ by $\sin \theta_{1} \equiv r_{1} / r_{2}$, and using the convention $\mu \equiv \cos \theta$ (thus $\mu_{1}=\cos \theta_{1}$ ), the optical depth is given by

$$
\tau(\theta)=\left\{\begin{array}{lr}
2 \tau_{2} \mu & \text { if } \mu \leq \mu_{1} \\
2 \tau_{2}\left(\mu-\sqrt{\mu^{2}-\mu_{1}^{2}}\right) & \mu>\mu_{1}
\end{array} .\right.
$$

The emerging luminosity from the surface of the sphere $\left(r=r_{2}\right)$ is

$$
\mathcal{L}_{\nu}=4 \pi r_{2}^{2} F_{\nu}=4 \pi r_{2}^{2} \oint I_{\nu}(\mu) \mu d \mu d \phi
$$

where $\phi$ is the angle in the plane perpendicular to the line of sight. Since we are considering isotropic, spherical emission, $I_{\nu}$ is independent of $\phi$, and since there is only vacuum contributing to rays coming from $\pi / 2 \leq \theta \leq \pi$,

$$
\mathcal{L}_{\nu}=2 \pi\left(4 \pi r_{2}^{2}\right) \int_{0}^{1} I_{\nu}(\mu) \mu d \mu=\frac{L_{\nu, \operatorname{thin}} 4 \pi r_{2}^{2}}{2 V \alpha} \int_{0}^{1}\{1-\exp [-\tau(\mu)]\} \mu d \mu .
$$

We here observe that the volume-to-surface-area can be used to define a characteristic width of the shell,

$$
\widetilde{\Delta r} \equiv \frac{V}{4 \pi r_{2}^{2}}=r_{2} \frac{1}{3}\left[1-\left(\frac{r_{1}}{r_{2}}\right)^{3}\right],
$$

so the escape fraction is

$$
\frac{\mathcal{L}_{\nu}}{\mathcal{L}_{\nu, \text { thin }}}=\frac{1}{2 \alpha \widetilde{\Delta r}} \int_{0}^{1}\left(1-e^{-\tau}\right) \mu d \mu
$$

Here, $\mathcal{L}_{\nu, \text { thin }}$ is the luminosity the gas would have if it were optically thin. In general, given the form of $\tau(\theta)$ (Equation A3), this integral must be computed numerically. In the limit $\tau_{2} \ll 1$, this integral recovers $\mathcal{L}_{\nu}=\mathcal{L}_{\nu \text {, thin }}$ (we note for the reader's convenience in checking this result themselves that when evaluating the optically thin limit, it is helpful to define a factor $f_{V}=\widetilde{\Delta r} / r_{2}$ and use $\left.\alpha V=\tau_{2} f_{V}\right)$. In the limit of high optical depth, the escape fraction is $\mathcal{L}_{\nu} / \mathcal{L}_{\nu, \text { thin }}=1 /(4 \alpha \widetilde{\Delta r})$.

The shell solution has the same asymptotic behaviors as the slab approximation, $\mathcal{L}_{\nu} / \mathcal{L}_{\nu \text {,thin }}=[1-\exp (-\tau)] / \tau$, if one uses $\tau=4 \alpha \widetilde{\Delta r}$. We find that the error on the escape fraction incurred by using the slab approximation versus numerical integration depends on the thickness of the emitting region $\left(r_{1} / r_{2}\right)$ and $\alpha \widetilde{\Delta r}$ but in any case is $<10 \%$. The error is highest for thin shells $\left(r_{1} / r_{2} \gtrsim 0.8\right)$ and near the transition between optically thick and thin regimes $(\alpha \widetilde{\Delta r} \sim 0.5)$. Therefore, we consider a slab approximation to be suitable in this work, and use

$$
\frac{\mathcal{L}_{\nu}}{\mathcal{L}_{\nu, \text { thin }}}=\frac{1-\exp (-4 \alpha \widetilde{\Delta r})}{4 \alpha \widetilde{\Delta r}}
$$

\section{A.2. Including Absorption by an External Medium}

In this scenario we have the same emitting (and self-absorbing) shell as in the last case, but additionally there is absorption from an external shell that extends from $r_{2}$ (the edge of the emission region) to $r_{3}$ (the edge of the CSM shell), representing the as-yet-unshocked CSM.

The specific intensity along any path is

$$
I_{\nu}(z)=\frac{\mathcal{L}_{\nu, \text { thin }}}{4 \pi \alpha V}\left(1-\exp \left[-\tau_{\mathrm{ssa}}(z)\right]\right) \exp \left[-\tau_{\mathrm{ff}}(z)\right],
$$

where $z$ is the height above the equator, $\tau_{\mathrm{ssa}}$ is the optical depth to synchrotron self-absorption (internal absorption; simply called " $\tau$ " in the previous calculation), $\tau_{\mathrm{ff}}$ is the optical depth to free-free absorption (external absorption), and all other variables are as before. We use $z$ rather than $\theta$ here because in terms of $z$ the integral to calculate flux 
has the same limits with the external absorption as without. To maintain the definition of $\theta$ as the angle relative to the surface normal at $r_{2}$, we define $\theta^{\prime}$ to be the angle relative to the surface normal at $r_{3}$; then $z=r_{2} \sin \theta=r_{3} \sin \theta^{\prime}$, and $\mu d \mu=-r_{2}^{-2} z d z=-r_{3}^{-2} z d z$. For this calculation, we are evaluating the flux at $r_{3}$ rather than $r_{2}$. Then

$$
\begin{aligned}
L_{\nu} & =4 \pi r_{3}^{2} \frac{L_{\nu, \text { thin }}}{2 \alpha V} \int_{0}^{r_{2}}\left[1-e^{-\tau_{\mathrm{ssa}}(z)}\right] e^{-\tau_{\mathrm{ff}}(z)} \frac{z d z}{r_{3}^{2}} \\
\frac{L_{\nu}}{L_{\nu, \text { thin }}} & =4 \pi \frac{1}{2 \alpha V} \int_{0}^{r_{2}}\left[1-e^{-\tau_{\mathrm{ssa}}(z)}\right] e^{-\tau_{\mathrm{ff}}(z)} z d z \\
& =\frac{1}{2 r_{2}^{2} \alpha \widetilde{\Delta r}} \int_{0}^{r_{2}}\left[1-e^{-\tau_{\mathrm{ssa}}(z)}\right] e^{-\tau_{\mathrm{ff}}(z)} z d z .
\end{aligned}
$$

Defining $\zeta=z / r_{2}$,

$$
\frac{L_{\nu}}{L_{\nu, \text { thin }}}=\frac{1}{2 \alpha \widetilde{\Delta r}} \int_{0}^{1}\left[1-e^{-\tau_{\mathrm{ssa}}(\zeta)}\right] e^{-\tau_{\mathrm{ff}}(\zeta)} \zeta d \zeta
$$

This is the exact solution for the escape fraction.

In this work we have approximated this result by simply accounting for external absorption with an exponential factor such that

$$
\frac{\mathcal{L}_{\nu}}{\mathcal{L}_{\nu, \text { thin }}}=\frac{1-\exp (-4 \alpha \widetilde{\Delta r})}{4 \alpha \widetilde{\Delta r}} \exp \left(-\alpha_{\mathrm{ff}} \Delta r_{\mathrm{ext}}\right)
$$

where $\Delta r_{\text {ext }}=r_{3}-r_{2}$ is the radial width of the CSM and $\alpha_{\mathrm{ff}}$ is the free-free (Bremsstrahlung) extinction coefficient, which is assumed to be constant in the preshock CSM.

In a case where the SSA optical depth is low, we computed the difference between the result of the numerical integral and this approximation for various values of $\alpha_{\mathrm{ff}} r_{3}$ (optical depth) and $r_{2} / r_{3}$ (absorbing medium thickness). We find that the error of the approximation increases as this $\alpha_{\mathrm{ff}} r_{3}$ increases, and that the error due to geometric effects is largest at $r_{2} / r_{3} \sim 0.5$. However, even for this worst case thickness, the error is $\sim 10 \%$ at $\tau \sim 1$ (approximation gives 10\% higher luminosity), and 100\% at $\tau \sim 10$ (approximation gives twice the luminosity). The error increases by approximately a decade for each increasing decade in $\tau$, but, in our view, it does not matter because the luminosity is essentially completely absorbed in this regime.

\section{REFERENCES}

Astropy Collaboration, Robitaille, T. P., Tollerud, E. J., et al. 2013, A\&A, 558, A33, doi: 10.1051/0004-6361/201322068

Botyánszki, J., Kasen, D., \& Plewa, T. 2018, ApJL, 852, L6, doi: 10.3847/2041-8213/aaa07b

Branch, D., Livio, M., Yungelson, L. R., Boffi, F. R., \&

Baron, E. 1995, PASP, 107, 1019, doi: 10.1086/133657

Cendes, Y., Drout, M. R., Chomiuk, L., \& Sarbadhicary,

S. K. 2020, ApJ, 894, 39, doi: 10.3847/1538-4357/ab6b2a

Chevalier, R. A. 1982, ApJ, 259, 302, doi: 10.1086/160167

Chomiuk, L., Linford, J. D., Yang, J., et al. 2014, Nature, 514, 339, doi: 10.1038/nature13773

Chomiuk, L., Soderberg, A. M., Chevalier, R. A., et al. 2016, ApJ, 821, 119, doi: 10.3847/0004-637X/821/2/119

Dilday, B., Howell, D. A., Cenko, S. B., et al. 2012, Science, 337, 942, doi: 10.1126/science.1219164
Fisher, R., \& Jumper, K. 2015, ApJ, 805, 150, doi: 10.1088/0004-637X/805/2/150

Graham, M. L., Kumar, S., Hosseinzadeh, G., et al. 2017, MNRAS, 472, 3437, doi: 10.1093/mnras/stx2224

Graham, M. L., Harris, C. E., Nugent, P. E., et al. 2019, ApJ, 871, 62, doi: 10.3847/1538-4357/aaf41e

Harris, C. E., Nugent, P. E., \& Kasen, D. N. 2016, ApJ, 823, 100, doi: 10.3847/0004-637X/823/2/100

Harris, C. E., Nugent, P. E., Horesh, A., et al. 2018, ApJ, 868, 21, doi: 10.3847/1538-4357/aae521

Hunter, J. D. 2007, Computing in Science \& Engineering, 9, 90, doi: 10.1109/MCSE.2007.55

Jones, E., Oliphant, T., Peterson, P., et al. 2001, SciPy: Open source scientific tools for Python. http://www.scipy.org/

Kasen, D., Thomas, R. C., \& Nugent, P. 2006, ApJ, 651, 366, doi: 10.1086/506190 
Kollmeier, J. A., Chen, P., Dong, S., et al. 2019, MNRAS, 486, 3041, doi: 10.1093/mnras/stz953

Leloudas, G., Hsiao, E. Y., Johansson, J., et al. 2015, A\&A, 574, A61, doi: 10.1051/0004-6361/201322035

Livio, M., \& Riess, A. G. 2003, ApJL, 594, L93, doi: $10.1086 / 378765$

Lundqvist, P., Kundu, E., Pérez-Torres, M. A., et al. 2020, ApJ, 890, 159, doi: 10.3847/1538-4357/ab6dc6

Mooley, K. P., Fender, R. P., Staley, T., et al. 2016, The Astronomer's Telegram, 8937, 1

Moore, K., \& Bildsten, L. 2012, ApJ, 761, 182, doi: 10.1088/0004-637X/761/2/182

Oliphant, T. 2006, A guide to NumPy. http://www.numpy.org/

Pellegrino, C., Howell, D. A., Sarbadhicary, S. K., et al. 2020, arXiv e-prints, arXiv:2003.05946. https://arxiv.org/abs/2003.05946

Pérez-Torres, M. A., Lundqvist, P., Beswick, R. J., et al. 2014, ApJ, 792, 38, doi: 10.1088/0004-637X/792/1/38

Perlmutter, S., Aldering, G., Goldhaber, G., et al. 1999, ApJ, 517, 565, doi: 10.1086/307221
Prieto, J. L., Chen, P., Dong, S., et al. 2020, ApJ, 889, 100, doi: 10.3847/1538-4357/ab6323

Riess, A. G., Filippenko, A. V., Challis, P., et al. 1998, AJ, 116, 1009, doi: 10.1086/300499

Roth, N., \& Kasen, D. 2015, ApJS, 217, 9, doi: 10.1088/0067-0049/217/1/9

Rybicki, G. B., \& Lightman, A. P. 1979, Radiative processes in astrophysics

Ryder, S., Kundu, E., Marnoch, L., Chomiuk, L., \& Sarbadhicary, S. 2019, The Astronomer's Telegram, 12820,1

Silverman, J. M., Nugent, P. E., Gal-Yam, A., et al. 2013, ApJS, 207, 3, doi: 10.1088/0067-0049/207/1/3

Tucker, M. A., Shappee, B. J., Vallely, P. J., et al. 2020, MNRAS, 493, 1044, doi: 10.1093/mnras/stz3390

van Hoof, P. A. M., Williams, R. J. R., Volk, K., et al. 2014, MNRAS, 444, 420, doi: 10.1093/mnras/stu1438

Weiler, K. W., Panagia, N., Montes, M. J., \& Sramek, R. A. 2002, ARA\&A, 40, 387, doi: 10.1146/annurev.astro.40.060401.093744

Weiler, K. W., Panagia, N., \& Sramek, R. A. 1990, ApJ, 364, 611, doi: 10.1086/169444

Wood-Vasey, W. M., Wang, L., \& Aldering, G. 2004, ApJ, 616, 339, doi: $10.1086 / 424826$ 